\title{
Pre-Transplant Therapy in Experimental Heart Transplantation
}

\begin{abstract}
Tomislav Stojanovic ${ }^{1}$, Andreas H. Wagner'2, Friedrich A. Schöndube ${ }^{1}$ and Markus Hecker ${ }^{2}$ ${ }^{1}$ Department of Cardiovascular and Thoracic Surgery, University of Göttingen, Göttingen, 2Institute of Physiology and Pathophysiology, Division of Cardiovascular Physiology, University of Heidelberg, Heidelberg,
\end{abstract}

Germany

\section{Introduction}

Heart transplantation is a life-saving procedure for patients with end-stage cardiac dysfunction. Currently one-year graft survival ranges between $77-88 \%$, three-year survival between $77-79 \%$ and five-year survival between $67-73 \%$ (http://www.americanheart.org/presenter.jhtml?identifier=4588). Despite these encouraging results, primary graft failure accounts for about $23 \%$ of deaths in the first 90 days post transplantation. Pretransplant variables associated with primary graft failure include: ischemia time, donor gender, donor age, multiorgan donation, center volume, extracorporeal membrane oxygenation, mechanical circulatory support, etiology of heart failure, and reoperative heart transplantation (Russo et al., 2010). To target rejection of allografts, a variety of immunosuppressive protocols are used solely in the recipient who is the main focus of the therapy (The International Society of Heart and Lung Transplantation Guidelines for the Care of Heart Transplant Recipients Task Force 2: Immunosuppression and Rejection (Nov. 8, 2010)). Typically, neither donor nor grafts are specifically treated before or during organ harvesting except for the maintenance of circulatory and respiratory functions of the donor and irrigation of grafts with preservation solutions.

\section{Graft rejection}

A T-cell driven process mediated by the adaptive immune system is our classic understanding of graft rejection. Allogeneic MHC molecules on graft tissue are recognized by the recipient's immune system by two pathways, the direct and the indirect pathway. Alloreactive T-cells in the direct pathway recognize donor MHC molecules on antigenpresenting cells (APCs) which pass through the transplanted tissues (Larsen et al., 1990). APCs in the indirect pathway process antigens which are derived from donor MHC molecules and present them to alloreactive T-cells (Game \& Lechler, 2002). Their activation is further fuelled by co-stimulatory molecules such as the CD40-CD40 ligand (CD154) receptor-ligand pathway (Cho et al., 2000). This co-stimulation, in case of graft endothelial cells, not only reinforces T-cell activity but enhances the later recruitment of effector leukocytes, namely monocytes (Rose, 1997). Leukocyte-endothelial cell interaction, thus 
exaggerated, causes damage to the capillary microcirculation, organ dysfunction and ultimately graft loss (Cho et al., 2000; Rocha et al., 2003).

Although T-cells are believed to play a critical role in acute rejection, it could be shown that expression of pro-inflammatory mediators within the allograft is up-regulated even before the T-cell response. This response is mediated by the innate immune system which is independent of the adaptive immune reaction (Christopher et al., 2002; He et al., 2002; He et al., 2003). By means of RNase protection assay in a mouse heart transplantation model it has been shown that one day after transplantation the gene expression profile of the cellular infiltrate, namely chemokine receptor expression and the expression of pro-inflammatory cytokines, was similar in RAG-deficient mice as compared to immunologically competent wild type mice (He et al., 2002). It could therefore be concluded that innate, antigenindependent pro-inflammatory processes occur shortly after transplantation and are further specifically modified by the adaptive immune response (He et al., 2003).

\section{Innate immune system and ischemia reperfusion in organ transplantation}

Multicellular organisms have developed immunological mechanisms to detect and to rapidly respond to threats. Such immunological mechanisms are being referred to as innate immunity. Unlike the evolutionary younger adaptive immune system, the innate immune system performs its control function by using so-called "pattern recognition" receptors (PRRs) (Janeway \& Medzhitov, 2002). PRRs identify pathogenic molecules, microbial "nonself" (pathogen-associated molecular patterns [PAMP]) or recipient molecules released from damaged or "stressed" tissues ("damaged self" or damage-associated molecular patterns [DAMP]) that differ from "self" which are thought to signal through PRRs such as toll-like receptor 4 (TLR4) in a manner similar to PAMPs (Mollen et al., 2006).

Different cell types play a role in the effector response, among others dendritic cells, natural killer cells and endothelial cells. They detect PAMPs as exogenous and DAMPs as endogenous ligands through toll-like receptors (Land, 2003; Land, 2005; Takeda \& Akira, 2005) (Figure 1).

Engagement of DAMPs with TLRs leads to activation of transcription factors STAT-1, AP-1, NF- $\kappa \mathrm{B}$ and IRF-3 followed by chemokine (e.g. MCP-1) and pro-inflammatory cytokine release, graft damage and ultimately graft loss.

After recognition of PAMPs the activated TLRs initiate an intracellular signalling cascade which leads to activation of transcription factors such as, e.g. activator protein-1 (AP-1), nuclear factor- $\mathrm{KB}$ (NF-KB) or interferon regulatory factor-3 (IRF-3), expression of proinflammatory genes and, e.g. maturation of dendritic cells (Ishitani et al., 2003; Hemmi \& Akira, 2005; Kishida et al., 2005; Sato et al., 2005; Shim et al., 2005; Takeda \& Akira, 2005). Mature dendritic cells interact with naïve T-cells and trigger a T-cell response. They act therefore as a link between innate and adaptive immunity.

The process of organ injury starts even before the recipient's immune system has the first contact with donor cells. Its starts with the oxidative damage to the graft in the brain-dead donor and continues after reperfusion (Land, 2005). NADPH oxidase-dependent reactive oxygen species (ROS) production is involved in translocation of TLR4 to lipid rafts (Nakahira et al., 2006), i.e. micro-domains of the cell membrane, a process that is important 


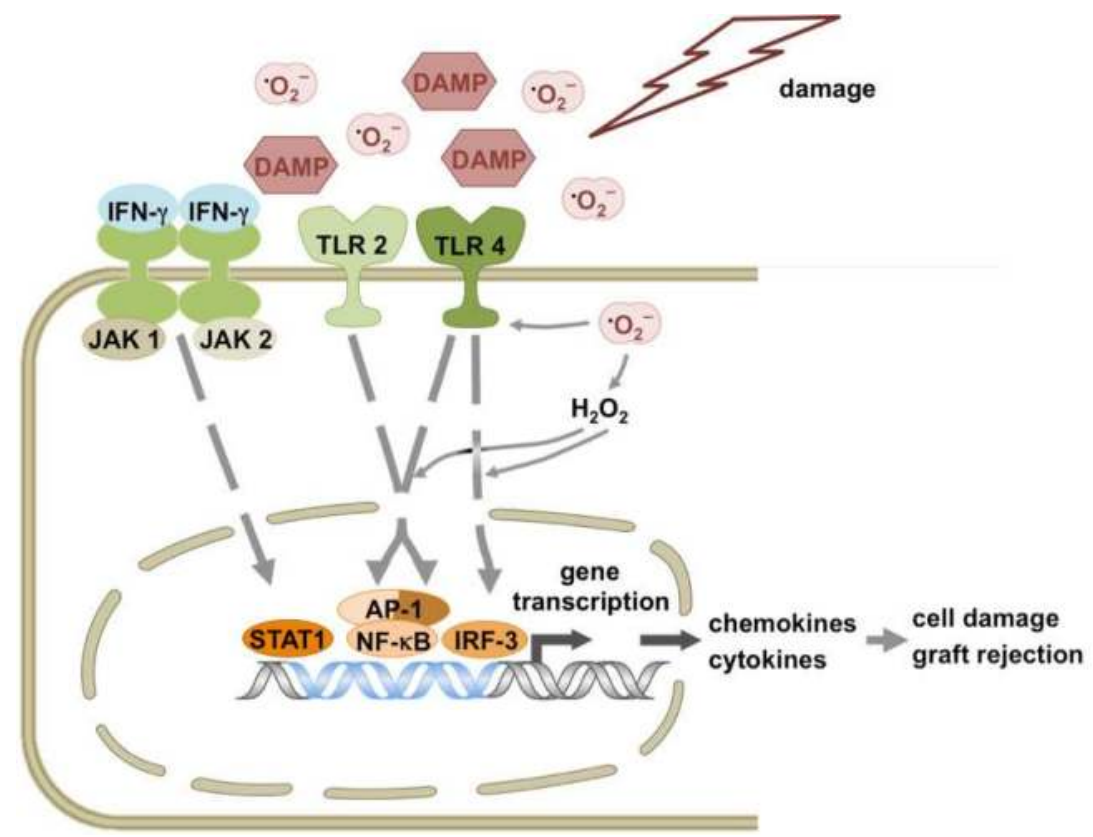

Fig. 1. Scheme of the innate immunity effector response after challenge by DAMPs (modified after Land 2007 (Land, 2007)).

in signal transduction (Dykstra et al., 2003; Manes et al., 2003). DAMPs like heat shock protein 72 (HSP 72), high mobility group box 1 (HMGB-1) and fragments of hyaluronic acid (fHA) are recognised by TLR4 and/or TLR2 (Land, 2007). Activation of these TLRs initiates a signal transduction cascade to the nucleus through molecules like myeloid differentiation marker 88 (MyD88), TIR-associated protein (TIRAP) und TIR domain-containing adaptor inducing IFN- $\beta$ (TRIF), ultimately leading to activation of the transcription factors AP-1, NF$\mathrm{kB}$ or IRF-3, up-regulation of pro-inflammatory gene expression and, among others, maturation of dendritic cells that all fuel the immunologic reaction against the graft (Land, 2007).

In summary, ROS in the brain-dead donor and during reperfusion cause i) initial graft damage which activates the innate immune response, ii) activation of signalling pathways which further augment this response and iii) boosting of humoral, complement-mediated tissue damage (Land, 2007).

\section{Graft rejection and endothelial cells}

In the setting of graft rejection, endothelial cells are the first donor cells which are affected by activation of the innate and adaptive immune response of the recipient. Integrity of the donor endothelium is of pivotal importance to graft function because injury to the endothelium ultimately leads to perfusion failure and graft loss. In addition, the endothelial cells themselves activate the innate immune response thereby maintaining a vicious circle (Rose, 1997; Rose, 1998; Methe et al., 2007). Graft injury due to ROS and activation of the 
innate immune response occurs very early at the level of the donor endothelial cells. In this context, systemic immunosuppressive therapy has the disadvantage of being initiated in the recipient while graft damage is already in transition and furthermore it does not specifically target endothelial cell damage. In fact, it may even augment it. Therefore, an adjunct pretransplant therapy solely within the graft that addresses the pro-inflammatory effects of the innate immune response on the endothelium seems to hold great promise. Moreover, by excluding systemic effects, the therapy can be adequately dosed and administered directly to the target site because the graft vessels are readily accessible after organ explantation.

\section{Decoy oligodeoxynucleotide technology in organ transplantation}

To specifically address this setting, the decoy oligodeoxynucleotide (ODN) technology may provide a specific tool to directly interfere with target gene expression. Decoy (decoy=bait) ODNs are short double-stranded DNA molecules with a specific sequence of bases that typically matches the consensus DNA recognition motif of the target transcription factor. With these nucleic acid-based drugs it is possible to effectively inhibit the expression of genes which are controlled by the said transcription factor (Morishita et al., 1995). Most mammalian cells are easily transfected with these DNA molecules. In fact, their uptake by most target cells does not require any transfection agent or the like, and under physiological conditions is mediated by active transport, i.e. a carrier system and/or receptor mediated endocytosis (Figure 2).

The synthetic, 15 to $25 \mathrm{bp}$ long DNA fragments usually are stabilized by replacing 2 to 3 of the terminal phosphodiester bonds between the nucleotides by phosphothio-ester bonds

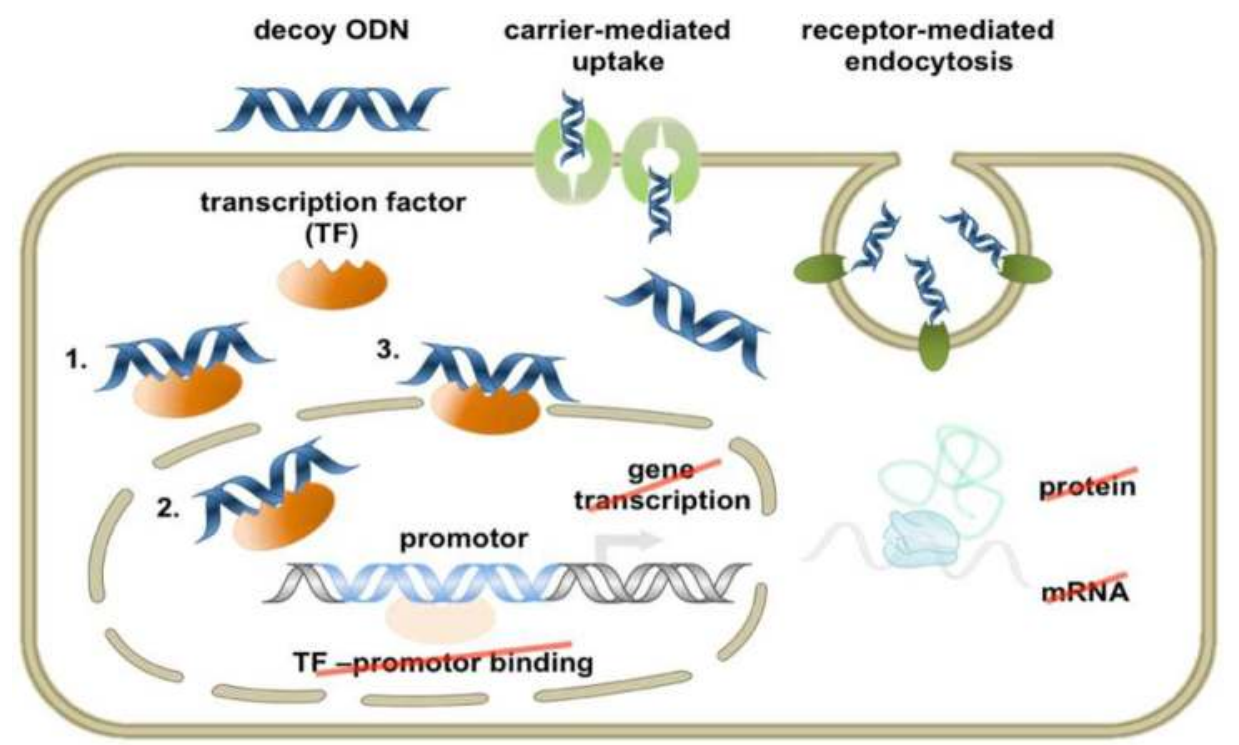

Fig. 2. Decoy ODN mechanism of action. Scheme depicting the mechanism of action of decoy ODN-mediated neutralization of the targeted transcription factor (TF). 1 refers to cytoplasmic TF sequestration, 2 to nuclear TF sequestration, and 3 to removal of promotorbound TF through titration with high-affinity decoy ODNs. 
that afford remarkable protection against exonucleases so that they remain intact in the target cells for some days. As mentioned above, decoy ODNs typically harbour at least one binding site for a particular transcription factor or transcription factor family. The length of this binding site normally is derived from known DNA binding motifs for the target transcription factor in different species and on the basis of their homology (consensus sequence).

Following their uptake by the respective target cell(s), decoy ODNs bind to their target transcription factor with high affinity (comparable to the formation of an antigen-antibody complex) thereby preventing it from translocation to the nucleus and/or effectively neutralizing its activity therein.

\section{Role of interferon- $\gamma$ and signal transducer and activator of transcription-1 (STAT-1) in transplant rejection}

Acute cellular rejection reduces the short and long-term outcome after transplantation. Recipient immune cells are recruited from the circulation into the transplant by presentation of antigens to T-helper cells whose activation is further increased by co-stimulatory molecules such as the CD40-CD154 receptor-ligand pathway (Cho et al., 2000). As a consequence, activated T-helper cells induce the increased expression of adhesion molecules on the endothelial cells of the donor organ, which leads to further recruitment of circulating monocytes into the graft and ultimately graft failure (Cho et al., 2000).

An important cytokine that is produced by activated T-helper cells is interferon- $\gamma$ (IFN- $\gamma$ ). Due to activation of the transcription factor signal transducer and activator of transcription1 (STAT-1) IFN- $\gamma$ up-regulates the expression of a number of important genes for graft rejection in the endothelial cells of the donor organ such as, e.g. vascular cell adhesion molecule-1 (VCAM-1) or CD40 (De Caterina et al., 2001; Wagner et al., 2002). VCAM-1 expression, for example, fuels leukocyte-endothelial cell interaction which is a hallmark of acute vascular rejection (De Caterina et al., 2001; Wagner et al., 2002).

Signalling of IFN- $\gamma$ to the nucleus of an endothelial cell starts with its binding to the corresponding receptor on the cell surface which then dimerizes (Bach et al., 1997; Boehm et al., 1997; Pestka, 1997). As a consequence, the receptor-associated tyrosine kinases JAK1 and JAK2, members of the Janus family of kinases (Boehm et al., 1997; Pestka, 1997), translocate to the cell membrane. There they phosphorylate the cytoplasmic domain of the IFN- $\gamma$ receptor which is now able to recruit monomeric STAT-1 (Ivashkiv, 1995; Ihle, 1996; Darnell, 1997). After phosphorylation and dimerization the now active transcription factor translocates to the nucleus and stimulates IFN- $\gamma$-dependent gene expression by binding to the gamma-interferon-activated element (GAS) in the promoter region of its target genes (Ivashkiv, 1995; Ihle, 1996; Darnell, 1997). In addition to CD40, the expression of ICAM-1 (Naik et al., 1997) or that of the inducible isoform of nitric oxide (NO) synthase is also controlled by STAT-1 (Ganster et al., 2001).

IFN- $\gamma$ as a multifunctional cytokine therefore controls many pro-inflammatory gene products whose expression is stimulated through the IFN- $\gamma /$ STAT-1 pathway (Khabar et al., 2004; Khabar \& Young, 2007; Liu et al., 2008; Karonitsch et al., 2009). In vivo and in vitro experiments showed that the up-regulation of both CD40 and VCAM-1 expression by STAT1 is effectively reduced using decoy ODNs directed against this transcription factor (Wagner et al., 2002; Quarcoo et al., 2004). 
The role of CD40-CD154 co-stimulation in transplant rejection still is the subject of intense research (Larsen \& Pearson, 1997; Cho et al., 2000). Thus it was shown in human biopsies of heart transplants that there is a significant correlation between the increased expression of CD154 and CD40 on leukocytes and endothelial cells, respectively, and acute rejection (Reul et al., 1997). The administration of monoclonal antibodies directed against CD154 prolonged renal transplant survival in rats and primates (Gudmundsdottir \& Turka, 1999; Hancock, 1999; Kirk et al., 1999). Furthermore, the application of anti-CD40 antibodies in monkeys led to a significant immunosuppressive effect and prolonged graft survival (Imai et al., 2007), so that both CD40 and CD154 can be regarded as important target molecules in allograft rejection. The clinical development of the corresponding biological (humanized monoclonal anti-CD154) antibodies, however was previously thwarted by serious thromboembolic complications (Kawai et al., 2000; Koyama et al., 2004), which are suspected to have been caused by an interference with the stabilizing effect of soluble CD154 on freshly formed thrombi (Andre et al., 2002). In fact, the main source of soluble CD154 are aggregating platelets from which the membrane-bound CD154 is shed upon activation (Kaya et al., 2011; Pamukcu et al., 2011; Silvain et al., 2011; Yacoub et al., 2010). More recently, however, some progress has been made towards the development of monoclonal antibodies with less side effects (Hussein et al., 2010).

CD40 is a cell surface receptor that belongs to the family of tumor necrosis factor receptors. It is mainly expressed on B-cells and antigen-presenting cells, but also on other non-immune cells such as vascular smooth muscle cells, fibroblasts and endothelial cells (van Kooten \& Banchereau, 2000; Schonbeck \& Libby, 2001). The corresponding ligand, CD154, is a member of the TNF family and located on activated T-cells, dendritic cells, mast cells, eosinophils, activated platelets and endothelial cells (Cho et al., 2000). CD40 stimulation in endothelial cells elicits a marked increase in expression of adhesion molecules and chemokines, which in turn facilitate the extravasation of leukocytes and their accumulation in the inflamed tissue (Hollenbaugh et al., 1995; Yellin et al., 1995). In addition, endothelial cells, as APCs, produce interleukin-12 (IL-12) in response to CD40 stimulation, which may play a crucial role in strengthening the T-cell response during graft rejection (Ode-Hakim et al., 1996; D'Elios et al., 1997). This IL-12 production is strongly enhanced in the presence of IFN- $\gamma$.

\section{STAT-1 decoy ODN treatment to ameliorate cardiac allograft survival}

Since targeting CD154 therapeutically appears to be wrought with difficulties, CD40 may be the more suitable drug target. However, low-molecular-weight CD40 antagonists are not yet available and CD40 antibodies, although prolonging graft survival in earlier studies, may cause anti-antibody formation and anaphylaxis (Imai et al., 2007). Therefore, an alternative approach was sought addressing transcription of the CD40 gene in endothelial cells, and this was achieved by employing decoy ODNs directed against the transcription factor STAT-1.

To investigate this novel therapeutic concept in terms of acute and chronic graft rejection, STAT-1 decoy ODNs were applied in established models of allogeneic cardiac transplantation in rats and mice with or without immunosuppression and in both acute and chronic settings (Holschermann et al., 2006; Stadlbauer et al., 2008; Stojanovic et al., 2009). The exclusive uptake of the decoy ODNs by the donor coronary endothelium could be confirmed by administration of fluorescent dye-labelled nucleic acids. Given the pleiotropic influence of 
CD154/CD40 expression and of IFN- $\gamma$ on pro-inflammatory gene expression and the putative beneficial effect of STAT-1 decoy ODNs, the up-regulation of important chemokines and adhesion molecules was examined in cardiac allografts following periprocedural STAT-1 decoy ODN administration. To this end the expression of, e.g. CD40, VCAM-1, E-selectin, MCP-1, ICAM-1 and RANTES (Holschermann et al., 2006; Stojanovic et al., 2009) was analyzed. Furthermore, histological parameters of rejection and the profile of infiltrating inflammatory cells were monitored (Holschermann et al., 2006; Stadlbauer et al., 2008; Stojanovic et al., 2009).

As verified by real time PCR analysis, a single periprocedural application of STAT-1 decoy ODNs to the coronary arteries of allogeneic donor heart transplants resulted in a marked down-regulation of CD40, VCAM-1, E-selectin, MCP-1, ICAM-1 and RANTES expression in allografts but not in isografts 24 hours post transplantation when compared to allograft and isograft control hearts (Stojanovic et al., 2009). The corresponding mutant control ODNs showed no such effect on expression of these marker genes (Holschermann et al., 2006; Stojanovic et al., 2009).

In fact, expression of these gene products was even lower than in control syngeneic transplants. Moreover, in syngenic transplants, irrespective of the chosen treatment regimen, no change in the expression of CD40, ICAM-1 or MCP-1 was noted, but an increase in Eselectin and VCAM-1 expression occurred which probably is the consequence of a moderate surgical and ischemia/reperfusion trauma (Stojanovic et al., 2009). The expression of the aforementioned pro-inflammatory gene products in the allograft is IFN- $\gamma$-dependent and controlled by subsequent activation of the transcription factor STAT-1 (De Caterina et al., 2001; Burysek et al., 2002; Wagner et al., 2002). In addition, some of these pro-inflammatory gene products, e.g. MCP-1, regulate the expression of other pro-inflammatory genes (Saiura et al., 2004), so that a blockade of IFN- $\gamma$-induced MCP-1 expression through STAT-1 decoy ODN treatment probably could affect a much larger spectrum of pro-inflammatory gene products.

Besides MCP-1, which was still up-regulated 9 days after transplantation in the allografts compared with syngeneic transplanted hearts, there were no long-term changes in gene expression found in the donor hearts, so that the possible inhibitory effect of the STAT-1 decoy ODN treatment was no longer detectable at this time (Stojanovic et al., 2009).

When analyzing the profile of infiltrating cells, it was readily apparent that the number of infiltrating monocytes/macro-phages and T-cells was significantly reduced 6 days and 9 days post transplantation as compared to the allogeneic controls (Holschermann et al., 2006; Stojanovic et al., 2009). In line with these findings, the vascular and interstitial rejection scores were significantly reduced in STAT-1 decoy ODN-treated allografts 9 days post transplantation when compared to control allografts (Stojanovic et al., 2009), and accordingly a significantly prolonged graft survival was noted after single STAT-1 decoy ODN treatment in an allogeneic rat model (Holschermann et al., 2006).

This approach of specific interference with transcriptional regulation of gene expression by using decoy ODNs also holds true when targeting other key molecules involved in CD40 expression. Thus decoy ODNs directed against the transcription factor AP-1 also inhibited endothelial ICAM-1 and VCAM-1 expression and reduced the number of leukocytes, namely T-cells, infiltrating the graft blood vessels, and prolonged survival in acute rejection of cardiac allografts (Holschermann et al., 2006). 
Analyzing the course of chronic rejection after single application of either a STAT-1 or AP-1 decoy ODN in a rat cardiac transplantation model with immunosuppression revealed that after 100 days allografts treated with either decoy ODN showed a significantly lesser degree of disease and number of occluded vessels, pointing towards a profound inhibition of cardiac allograft vasculopathy (CAV) (Stadlbauer et al., 2008). As putative mechanism of action, it was proposed that the AP-1 decoy ODN primarily attenuated basal endothelial CD40 expression while the STAT-1 decoy ODN suppressed tumour necrosis factor- $\alpha / \mathrm{IFN}-\gamma$-stimulated expression of $\mathrm{CD} 40$ in the rat native endothelial cells (Stadlbauer et al., 2008).

In the setting of inflammatory disease in humans another or perhaps alternative mechanism of CD40 expression in endothelial cells may also be active. In human cultured endothelial cells, combined treatment with IFN- $\gamma$ and TNF- $\alpha$ induces an IRF-1-dependent de novo expression of CD40. Although this effect is indirectly mediated by STAT-1 through induction of IRF-1 synthesis, STAT-1 directly induces CD40 expression in these cells when exposed to IFN- $\gamma$ alone. Thus, it is the cytokine composition under the given proinflammatory conditions that determines which transcription factor is primarily responsible for the increase in endothelial CD40 expression (Wagner et al., 2002).

\section{Conclusion}

The primary injury inflicted on the graft before implantation actually takes place currently does not receive sufficient attention. The mechanisms of innate immunity, which are significantly contributing to primary graft damage, are also not well understood, but at least regained scientific interest.

Herein an attempt was made to highlight some consequences of the inadvertently exaggerated activation of the innate immune response in cardiac transplant rejection with special emphasis on the role of the donor endothelium and the underlying proinflammatory mechanisms. By blocking the expression of pro-inflammatory gene products in these cells beneficial effects on the perfusion of the graft and its histological appearance can be achieved. The tested animal experimental models did not comprise a conventional immunosuppressive treatment regimen with regard to acute rejection and despite the fully allogeneic transplantation setting. There is the distinct possibility therefore that combining conventional immunosuppressive therapy with the presented novel decoy ODN drug approach results in an adequate immunosuppression with much less toxicity and a significantly better outcome with respect to long-term transplant function and survival. We believe that the underlying mechanism of action of these nucleic acid-based drugs can spur a promising field of research with midterm clinical implementation as a procurable perspective.

\section{Acknowledgments}

The authors are indebted to Dr. Gerd König for providing the figures and for critically reading of the manuscript. They are supported by the German Research Foundation (DFG), the Federal Ministry of Education and Research (BMBF), the European Commission and the German Cardiac Society (DGK). 


\section{References}

Andre, P., K. S. Prasad, et al. (2002). "CD40L stabilizes arterial thrombi by a beta3 integrin-dependent mechanism." Nat Med 8(3): 247-52.

Azuma, H., L. C. Paul, et al. (1996). "Insights into acute and chronic rejection." Transplant Proc 28(4): 2081-4.

Bach, E. A., M. Aguet, et al. (1997). "The IFN gamma receptor: a paradigm for cytokine receptor signaling." Annu Rev Immunol 15: 563-91.

Boehm, U., T. Klamp, et al. (1997). "Cellular responses to interferon-gamma." Annu Rev Immunol 15: 749-95.

Burysek, L., T. Syrovets, et al. (2002). "The serine protease plasmin triggers expression of MCP-1 and CD40 in human primary monocytes via activation of p38 MAPK and janus kinase (JAK)/STAT signaling pathways." J Biol Chem 277(36): 33509-17.

Cho, C. S., M. M. Hamawy, et al. (2000). "CD40:CD154 interactions and allograft rejection." Current Opinion in Organ Transplantation 5: 10-15.

Christopher, K., T. F. Mueller, et al. (2002). "Analysis of the innate and adaptive phases of allograft rejection by cluster analysis of transcriptional profiles." J Immunol 169(1): 522-30.

D'Elios, M. M., R. Josien, et al. (1997). "Predominant Th1 cell infiltration in acute rejection episodes of human kidney grafts." Kidney Int 51(6): 1876-84.

Darnell, J. E., Jr. (1997). "STATs and gene regulation." Science 277(5332): 1630-5.

De Caterina, R., T. Bourcier, et al. (2001). "Induction of endothelial-leukocyte interaction by interferon-gamma requires coactivation of nuclear factor-kappaB." Arterioscler Thromb Vasc Biol 21(2): 227-32.

Dykstra, M., A. Cherukuri, et al. (2003). "Location is everything: lipid rafts and immune cell signaling." Annu Rev Immunol 21: 457-81.

Game, D. S. and R. I. Lechler (2002). "Pathways of allorecognition: implications for transplantation tolerance." Transpl Immunol 10(2-3): 101-8.

Ganster, R. W., B. S. Taylor, et al. (2001). "Complex regulation of human inducible nitric oxide synthase gene transcription by Stat 1 and NF-kappa B." Proc Natl Acad Sci U S A 98(15): 8638-43.

Gudmundsdottir, H. and L. A. Turka (1999). "T cell costimulatory blockade: new therapies for transplant rejection." J Am Soc Nephrol 10(6): 1356-65.

Hancock, W. W. (1999). "Current trends in transplant immunology." Curr Opin Nephrol Hypertens 8(3): 317-24.

He, H., J. R. Stone, et al. (2002). "Analysis of robust innate immune response after transplantation in the absence of adaptive immunity." Transplantation 73(6): 853-61.

He, H., J. R. Stone, et al. (2003). "Analysis of differential immune responses induced by innate and adaptive immunity following transplantation." Immunology 109(2): 18596.

Hemmi, H. and S. Akira (2005). "TLR signalling and the function of dendritic cells." Chem Immunol Allergy 86: 120-35.

Hollenbaugh, D., N. Mischel-Petty, et al. (1995). "Expression of functional CD40 by vascular endothelial cells." J Exp Med 182(1): 33-40.

Hölschermann, H., T. H. Stadlbauer, et al. (2006). "STAT-1 and AP-1 decoy oligonucleotide therapy delays acute rejection and prolongs cardiac allograft survival." Cardiovasc Res 71(3): 527-36. 
http:/ / www.americanheart.org/presenter.jhtml?identifier $=4588$.

Hussein, M., J. R. Berenson, et al. (2010). "A phase I multidose study of dacetuzumab (SGN40; humanized anti-CD40 monoclonal antibody) in patients with multiple myeloma." Haematologica 95(5): 845-8.

Ihle, J. N. (1996). "STATs: signal transducers and activators of transcription." Cell 84(3): 3314.

Imai, A., T. Suzuki, et al. (2007). "A novel fully human anti-CD40 monoclonal antibody, 4D11, for kidney transplantation in cynomolgus monkeys." Transplantation 84(8): 1020-8.

Ishitani, T., G. Takaesu, et al. (2003). "Role of the TAB2-related protein TAB3 in IL-1 and TNF signaling." Embo J 22(23): 6277-88.

Ivashkiv, L. B. (1995). "Cytokines and STATs: how can signals achieve specificity?" Immunity 3(1): $1-4$.

Janeway, C. A., Jr. and R. Medzhitov (2002). "Innate immune recognition." Annu Rev Immunol 20: 197-216.

Karonitsch, T., E. Feierl, et al. (2009). "Activation of the interferon-gamma signaling pathway in systemic lupus erythematosus peripheral blood mononuclear cells." Arthritis Rheum 60(5): 1463-71.

Kawai, T., D. Andrews, et al. (2000). "Thromboembolic complications after treatment with monoclonal antibody against CD40 ligand." Nat Med 6(2): 114.

Kaya, Z., K. Ozdemir, et al. (2011). "Soluble CD40 ligand levels in acute pulmonary embolism: a prospective, randomized, controlled study." Heart Vessels (DOI 10.1007/s00380-011-0142-4).

Khabar, K. S., L. Al-Haj, et al. (2004). "Expressed gene clusters associated with cellular sensitivity and resistance towards anti-viral and anti-proliferative actions of interferon." J Mol Biol 342(3): 833-46.

Khabar, K. S. and H. A. Young (2007). "Post-transcriptional control of the interferon system." Biochimie 89(6-7): 761-9.

Kirk, A. D., L. C. Burkly, et al. (1999). "Treatment with humanized monoclonal antibody against CD154 prevents acute renal allograft rejection in nonhuman primates." Nat Med 5(6): 686-93.

Kishida, S., H. Sanjo, et al. (2005). "TAK1-binding protein 2 facilitates ubiquitination of TRAF6 and assembly of TRAF6 with IKK in the IL-1 signaling pathway." Genes Cells 10(5): 447-54.

Koyama, I., T. Kawai, et al. (2004). "Thrombophilia associated with anti-CD154 monoclonal antibody treatment and its prophylaxis in nonhuman primates." Transplantation 77(3): 460-2.

Land, W. G. (2003). "Allograft injury mediated by reactive oxygen species: from conserved proteins of Drosophila to acute and chronic rejection of human transplants. Part III: Interaction of (oxidative) stress-induced heat shock proteins with Toll-like receptorbearing cells of innate immunity and its consequences for the development of acute and chronic allograft rejection." Transplantation Rev 17: 67-86.

Land, W. G. (2005). "The role of postischemic reperfusion injury and other nonantigendependent inflammatory pathways in transplantation." Transplantation 79(5): 50514. 
Land, W. G. (2007). "Innate immunity-mediated allograft rejection and strategies to prevent it." Transplant Proc 39(3): 667-72.

Land, W. G. (2007). "Innate immunity-mediated allograft rejection and strategies to prevent it." Transplant Proc 39(3): 667-72.

LaRosa, D. F., A. H. Rahman, et al. (2007). "The innate immune system in allograft rejection and tolerance." J Immunol 178(12): 7503-9.

Larsen, C. P., P. J. Morris, et al. (1990). "Migration of dendritic leukocytes from cardiac allografts into host spleens. A novel pathway for initiation of rejection." J Exp Med 171(1): 307-14.

Larsen, C. P. and T. C. Pearson (1997). "The CD40 pathway in allograft rejection, acceptance, and tolerance." Curr Opin Immunol 9(5): 641-7.

Le Moine, A., M. Goldman, et al. (2002). "Multiple pathways to allograft rejection." Transplantation 73(9): 1373-81.

Liu, X., L. Ye, et al. (2008). "Activation of the JAK/STAT-1 signaling pathway by IFNgamma can down-regulate functional expression of the MHC class I-related neonatal Fc receptor for IgG." J Immunol 181(1): 449-63.

Manes, S., G. del Real, et al. (2003). "Pathogens: raft hijackers." Nat Rev Immunol 3(7): 557-68.

Methe, H., S. Hess, et al. (2007). "Endothelial cell-matrix interactions determine maturation of dendritic cells." Eur J Immunol 37(7): 1773-84.

Mollen, K. P., R. J. Anand, et al. (2006). "Emerging paradigm: toll-like receptor 4-sentinel for the detection of tissue damage." Shock 26(5): 430-7.

Morishita, R., G. H. Gibbons, et al. (1995). "A gene therapy strategy using a transcription factor decoy of the E2F binding site inhibits smooth muscle proliferation in vivo." Proc Natl Acad Sci U S A 92(13): 5855-9.

Naik, S. M., N. Shibagaki, et al. (1997). "Interferon gamma-dependent induction of human intercellular adhesion molecule-1 gene expression involves activation of a distinct STAT protein complex." J Biol Chem 272(2): 1283-90.

Nakahira, K., H. P. Kim, et al. (2006). "Carbon monoxide differentially inhibits TLR signaling pathways by regulating ROS-induced trafficking of TLRs to lipid rafts." J Exp Med 203(10): 2377-89.

Ode-Hakim, S., W. D. Docke, et al. (1996). "Delayed-type hypersensitivity-like mechanisms dominate late acute rejection episodes in renal allograft recipients." Transplantation 61(8): 1233-40.

Pamukcu, B., G. Y. Lip. Et al., (2011). "The CD40-CD40L system in cardiovascular disease." Ann Med 43(5): 331-40.

Pestka, S. (1997). "The interferon receptors." Semin Oncol 24(3 Suppl 9): S9-18-S9-40.

Quarcoo, D., S. Weixler, et al. (2004). "Inhibition of signal transducer and activator of transcription 1 attenuates allergen-induced airway inflammation and hyperreactivity." J Allergy Clin Immunol 114(2): 288-95.

Reul, R. M., J. C. Fang, et al. (1997). "CD40 and CD40 ligand (CD154) are coexpressed on microvessels in vivo in human cardiac allograft rejection." Transplantation 64(12): 1765-74.

Rocha, P. N., T. J. Plumb, et al. (2003). "Effector mechanisms in transplant rejection." Immunol Rev 196: 51-64.

Rose, M. L. (1997). "Role of endothelial cells in allograft rejection." Vasc Med 2(2): 105-14. 
Rose, M. L. (1998). "Endothelial cells as antigen-presenting cells: role in human transplant rejection." Cell Mol Life Sci 54(9): 965-78.

Russo, M. J., A. Iribarne, et al. (2010) "Factors associated with primary graft failure after heart transplantation." Transplantation 90(4): 444-50.

Saiura, A., M. Sata, et al. (2004). "Antimonocyte chemoattractant protein-1 gene therapy attenuates graft vasculopathy." Arterioscler Thromb Vasc Biol 24(10): 1886-90.

Sato, S., H. Sanjo, et al. (2005). "Essential function for the kinase TAK1 in innate and adaptive immune responses." Nat Immunol 6(11): 1087-95.

Schönbeck, U. and P. Libby (2001). "The CD40/CD154 receptor/ligand dyad." Cell Mol Life Sci 58(1): 4-43.

Shim, J. H., C. Xiao, et al. (2005). "TAK1, but not TAB1 or TAB2, plays an essential role in multiple signaling pathways in vivo." Genes Dev 19(22): 2668-81.

Silvain, J., J. P. Collet, et al. (2011). "Composition of coronary thrombus in acute myocardial infarction." J Am Coll Cardiol 57(12): 1359-67.

Stadlbauer, T. H., A. H. Wagner, et al. (2008). "AP-1 and STAT-1 decoy oligodeoxynucleotides attenuate transplant vasculopathy in rat cardiac allografts." Cardiovasc Res 79(4): 698705.

Stojanovic, T., A. H. Wagner, et al. (2009). "STAT-1 decoy oligodeoxynucleotide inhibition of acute rejection in mouse heart transplants." Basic Res Cardiol 104(6): 719-29.

Takeda, K. and S. Akira (2005). "Toll-like receptors in innate immunity." Int Immunol 17(1): 114.

The International Society of Heart and Lung Transplantation guidelines for the care of heart transplant recipients Task Force 2:Immunosuppression and Rejection (Nov.8, www.ishlt.org/ContentDocuments/ISHLT_GL_TaskForce2_110810.pdf. from The International Society of Heart and Lung Transplantation guidelines for the care of heart transplant recipients Task Force 2:Immunosuppression and Rejection (Nov.8,2010), www.ishlt.org/ContentDocuments/ISHLT_GL_TaskForce2_110810.pdf.

van Kooten, C. and J. Banchereau (2000). "CD40-CD40 ligand." J Leukoc Biol 67(1): 2-17.

Wagner, A. H., M. Gebauer, et al. (2002). "Cytokine-inducible CD40 expression in human endothelial cells is mediated by interferon regulatory factor-1." Blood 99(2): 520-5.

Yacoub, D., A. Hachem, et al. (2010). "Enhanced levels of soluble CD40 ligand exacerbate platelet aggregation and thrombus formation through a CD40-dependent tumor necrosis factor receptor-associated factor-2/Rac1/p38 mitogen-activated protein kinase signaling pathway." Arterioscler Thromb Vasc Biol 30(12): 2424-33.

Yellin, M. J., J. Brett, et al. (1995). "Functional interactions of T cells with endothelial cells: the role of CD40L-CD40-mediated signals." J Exp Med 182(6): 1857-64. 


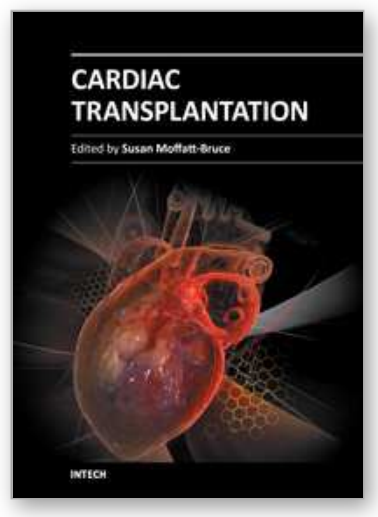

\author{
Cardiac Transplantation \\ Edited by Dr. Susan Moffatt-Bruce
}

ISBN 978-953-51-0000-3

Hard cover, 154 pages

Publisher InTech

Published online 10, February, 2012

Published in print edition February, 2012

We are truly in an era of change not only in terms of technology but in the type of patient we are caring for. That is why I feel this book is exciting in that it presents the team approach to the transplant patient. I am confident that the pioneers of cardiac transplantation would be pleased with our response to challenges in healthcare today and be pleased with the final product.

\title{
How to reference
}

In order to correctly reference this scholarly work, feel free to copy and paste the following:

Tomislav Stojanovic, Andreas H. Wagner, Friedrich A. Schöndube and Markus Hecker (2012). Pre-Transplant Therapy in Experimental Heart Transplantation, Cardiac Transplantation, Dr. Susan Moffatt-Bruce (Ed.), ISBN: 978-953-51-0000-3, InTech, Available from: http://www.intechopen.com/books/cardiactransplantation/preconditioning-therapy-in-experimental-heart-transplantation

\section{INTECH}

open science | open minds

\author{
InTech Europe \\ University Campus STeP Ri \\ Slavka Krautzeka 83/A \\ 51000 Rijeka, Croatia \\ Phone: +385 (51) 770447 \\ Fax: +385 (51) 686166 \\ www.intechopen.com
}

\author{
InTech China \\ Unit 405, Office Block, Hotel Equatorial Shanghai \\ No.65, Yan An Road (West), Shanghai, 200040, China \\ 中国上海市延安西路65号上海国际贵都大饭店办公楼 405 单元 \\ Phone: +86-21-62489820 \\ Fax: +86-21-62489821
}


(C) 2012 The Author(s). Licensee IntechOpen. This is an open access article distributed under the terms of the Creative Commons Attribution 3.0 License, which permits unrestricted use, distribution, and reproduction in any medium, provided the original work is properly cited. 